\title{
Competencias profesionales e investigación en Arteterapia
}

\author{
Miquel IZUEL I CURRI $\AA^{1}$ \\ miquelizuelcurria@gmail.com \\ Joan VALLÈS VILLANUEVA ${ }^{2}$ \\ joan.valles@udg.edu
}

Recibido: 30/05/12

Aceptado: 22/11/12

\section{RESUMEN}

Situar las competencias profesionales del arteterapeuta y específicamente su papel en la investigación en Arteterapia implica definir tanto el marco arteterapéutico donde va a desarrollar su acción como el conjunto de microcompetencias que, organizadas en un todo complejo, han de garantizar necesaria globalidad de las acciones y tareas a las que se enfrenta cualquier profesional.

Tanto la formación profesionalizadora como la formación permanente han de llevar a diseñar y gestionar procesos de investigación que permitan consolidar los avances, distribuir el conocimiento y mejorar las prácticas desarrolladas en Arteterapia.

Palabras clave: Arteterapia, marco arteterapéutico, profesión, investigación, competencias, metodologías, formación profesionalizadora, formación permanente.

\section{Referencia normalizada}

Izuel i Currià, M., Vallès Villanueva, J. (2012). "Competencias profesionales e investigación en Arteterapia”. En Arteterapia: Papeles de arteterapia y educación artística para la inclusión social Vol.7: páginas 13-26. Madrid. Servicio de publicaciones UCM.

\section{SUMARIO}

Introducción. El Arteterapia y la profesión de arteterapeuta. Una propuesta, Definición, Objetivos del Arteterapia, Marco Arteterapéutico, La posición ética y el rol del arteterapeuta. Competencias fundamentales como arteterapeuta y como investigador. Objetivos de la investigación en Arteterapia. Elementos y espacios para la investigación. Construyendo un paradigma de futuro. Bibliografía

\begin{abstract}
Locate the professional skills of the art-therapist and define specifically his/her role in research in Art Therapy involves defining both the art-therapeutic frame where he/she will develop his/her action and the micro-competencies whole that, organized into a complex whole, must ensure the necessary globality of the actions and tasks facing any professional.
\end{abstract}

\section{Professional skills and research in Art Therapy}

${ }^{1}$ Arteterapeuta y Psicoanalista. Presidente de la FEAPA (Federación Española de Asociaciones Profesionales de Arteterapia). Presidente de GREFART (Asociación Profesional de Arteterapeutas). Director del Master Integrativo en Arteterapia y del Diploma de Postgrado en Arteterapia de la Universitat de Girona (UdG)

${ }^{2}$ Coordinador del Master Interuniversitario Artes Visuales y Educación. Profesor del Master Integrativo en Arteterapia de la Universitat de Girona (UdG) 
All professionalizing training and lifelong learning have to lead to design and manage research processes that allow consolidating the gains, distributing knowledge and improving practices developed in Art Therapy.

Keywords: Art therapy, art-therapeutic framework, Profession, Research, Literacy, Methodologies, professional training, lifelong learning.

\section{CONTENTS}

Introduction. Art Therapy and the profession of art therapist. One proposal, Definition, Objectives of Art Therapy, Marco Arteterapéutico, The ethical position and the role of art therapist. Core competencies as art therapist and researcher. Research objectives in Art Therapy. Elements and spaces for research. Building a future paradigm. Bibliography

\section{INTRODUCCIÓN}

Cualquier disciplina necesita profesionales competentes y responsables de su permanente actualización profesional, que sean capaces de diseñar y gestionar procesos de investigación para así consolidar los avances, distribuir el conocimiento y mejorar las prácticas desarrolladas.

Todo profesional en Arteterapia ha de contemplar la necesidad ética de una interrogación permanente acerca de su práctica. Su formación teórica, técnica y práctico-metodológica ha de completarse con un proceso terapéutico personal, la supervisión de dicha práctica y la necesidad de potenciar las investigaciones y las publicaciones a fin de establecer procesos que permitan generar confianza, reconocimiento social y redes de conocimiento que contribuyan a la evaluación y la divulgación de los conocimientos relacionados con la disciplina. Por esta razón es necesario incorporar en la formación de las y los arteterapeutas propuestas que contribuyan al desarrollo de métodos científicos -adaptados al propio objeto que atañe al Arteterapia-, discusión sobre investigaciones realizadas y construcción de procesos críticos, sin interferir en la calidad de la práctica arteterapéutica que recibe su razón de ser desde la posición ética en que sitúa su trabajo.

De este modo debemos entender la investigación como un elemento imprescindible para atender adecuadamente las prácticas y el desarrollo de nuevos conocimientos y avances en la fundamentación de una ciencia en Arteterapia que beneficiará a todos los implicados pero principalmente a los destinatarios.

Como responsabilidad, las y los arteterapeutas deben ser capaces de evaluar e identificar las mejores evidencias de sus prácticas para que otros profesionales puedan también acceder a esos conocimientos y a su vez ayudar a la mejora de las competencias en Arteterapia.

Ya desde los dispositivos de formación inicial se debe atender esa necesidad y promover, en las y los alumnos, el interés por actitudes que permitirán el avance de la disciplina del Arteterapia como son:

- Cuestionarse los procesos seguidos en sus prácticas.

- Desarrollar procesos analíticos y críticos con el necesario rigor científico.

- Comprometerse en la permanente mejora de actividad profesional, desarrollando el espíritu investigador. 


\section{EL ARTETERAPIA Y LA PROFESIÓN DE ARTETERAPEUTA. UNA PROPUESTA \\ Definición}

El Arteterapia es una práctica que aborda la vivencia integradora de lo emocional (que para D. W. Winnicott implica a la capacidad de la persona de construir la realidad a partir de adaptarla a sus capacidades de creación). De ello se derivan una serie de efectos como son los psicoterapéuticos, de desarrollo del potencial humano y de la inclusión social. El principal objetivo tiende a que los usuarios se encuentren con la posibilidad de actualizar, a través del Arteterapia, sus capacidades de creación. Estas existen en la persona en un más allá de sus síntomas, edad, condición social y económica, cultura, origen, etc.

Se desarrolla en dos ejes:

- El de las capacidades creativas de actualizan en los procesos de creación que acontecen mientras se desarrollan producciones que pueden estar realizadas con el apoyo de múltiples lenguajes artísticos. Pintura, escultura, títeres, danza, escritura creativa, música, representaciones dramáticas, fotografía, performance, canto... Producciones que cuando se realizan conforman un proyecto vital de representaciones efectuadas en un itinerario que facilita el despliegue de los deseos, las inquietudes, los conflictos, los anhelos..., en un universo imaginativo. La persona va actualizando sus capacidades creadoras (re)creando, versionando su historia y restituyéndole su dimensión simbólica y poética. Todo de una forma cuidadosa que favorece la surgencia de las capacidades creativas, tanto para afrontar y resolver las dificultades como para desarrollar un proyecto vital de si mismo.

- El eje del vínculo es el desarrollado con el trabajo en la transferencia. El vínculo se va formando en la relación emocional entre el arteterapeuta y el usuario. El pasaje y resolución de la transferencia en el vínculo permite desarrollar nuevas formas de relaciones, tanto con los otros como con las partes menos conocidas de nosotros mismos.

Esta relación, atravesada por el respeto y la posición ética, facilita los cambios y las transformaciones deseadas. El trabajo en Arteterapia genera condiciones que facilitan poder reconocer las capacidades de creación y de curación que cada persona posee. Trabaja integralmente con la persona, con sus capacidades y deseos y no tan sólo con los síntomas y sus dificultades.

Se genera un lugar para los deseos, representados, traducidos, ubicados en un marco simbólico, donde la imaginación cobra su verdadero valor. Sin censura, invitando a realizar los cambios y las transformaciones que permiten desarrollar nuestra potencialidad de ser.

\section{Objetivos del Arteterapia}

Algunos objetivos fundamentales que debe atender la práctica del Arteterapia son los siguientes:

1. Promover una vivencia emocional integradora.

2. Actualizar las capacidades creativas. 
3. Acompañar en la construcción de nuevas dimensiones personales.

4. Otorgar formas de representación, con los soportes de los lenguajes artísticos, de las capacidades, de las dificultades y de sus formulaciones de resolución.

5. Desarrollar un lazo creativo a lo social y las funciones comunicativas pertinentes.

6. Acompañar el desarrollo de proyectos vitales.

\section{Marco Arteterapéutico}

El marco arteterapéutico visualiza y delimita la práctica del Arteterapia. Si antes hemos comentado y señalado los objetivos tal vez sea pertinente ahora decir que es lo que no atañe al Arteterapia como finalidad, aunque no sea desdeñable como beneficio asociado:

No se congratula con una mera expresión.

No busca el alivio sintomático.

No se usa como material proyectivo ni de diagnóstico.

No se contenta con una mera distracción.

No busca la rehabilitación.

El marco arteterapéutico ha de pensarse como un marco simbólico, contenedor, que delimita, asegura y sostiene. Protege y crea un espacio de confianza y de inter-juego. Se trata de una trama simbólica tejida con distintos vectores:

El tiempo, como soporte interno de la representación. Puede pensarse como dos vectores que actúan al unísono. Son el tiempo cronológico y el tiempo lógico:

- El cronológico está compuesto, a su vez, por los tiempos de la sesión y de la biografía. El de la sesión señala los tiempos para comenzar y para acabar el trabajo (día de encuentro, horario...) donde se despliega el itinerario de las resistencias y de la resolución creativa de las mismas. El biográfico refleja la inserción en el ciclo vital, restituye la dimensión histórica del sujeto.

- El tiempo lógico de cada una de las personas implicadas en la sesión, caracterizado por la atemporalidad del inconsciente que se manifiesta en el presente, el aquí y ahora que actualiza los contenidos de los complejos infantiles con toda su carga de actualidad coalescente.

El espacio, como soporte externo de la representación, donde la acción con la alteridad de los materiales se desarrolla haciendo posible la parte correspondiente del "construir la realidad adaptándola a las capacidades creativas". Un espacio que puede potenciar - por su exterioridad- la representación simbólica del sujeto. En él la persona figura y se configura con la producción. Espacio externo soporte y en diálogo con el espacio interno, fantasmatizado, "organizado" como si de escenas dramáticas se trataran, donde acontecen los diálogos.

La posición del arteterapeuta, que actúa como marco referencial, es soporte de las identificaciones. Representa la ley simbólica y por lo tanto está sometido a 
ella. Impele a la representación y no a la mera ilustración a partir de la escucha de las formas de la subjetividad en el otro.

Los materiales y las producciones, como elementos que, en su manipulación, preservan su alteridad radical, no dejándose atrapar totalmente por las proyecciones del psiquismo humano. Pueden estar en una relación más íntima -la voz- o más exterior -la plástica- con la persona, pero preservan la naturaleza de sus propias leyes. Materia con la que la persona dialoga para transformarla y transformarse en ese proceso, otorgándose campos de representación de si misma.

El vínculo, creación compartida y asimétrica, entre arteterapeuta y usuario. Trabajado en la transferencia, posibilita la actualización e integración de lo emocional.

El proceso arteterapéutico, itinerario no lineal -ni predecible- que da cuenta de las vicisitudes del trabajo arteterapéutico. En él se conjugan los vectores anteriormente mencionados.

Teniendo en cuenta lo anterior, la sesión de Arteterapia puede pensarse como un espacio potencial, donde el juego y la imaginación hacen de hilos conductores para el desarrollo de las capacidades creativas. Donde la producción puede operar como si de un objeto transicional se tratara y el arteterapeuta, parafraseando a D. Winnicott como una "madre suficientemente buena". Un espacio en suma, donde las vivencias poéticas y estéticas devienen experiencias emocionales.

\section{La posición ética y el rol del arteterapeuta}

Como toda intervención psicoterapéutica, el arteterapeuta recibe su autorización y función a partir de la persona que realiza la petición de ayuda, por lo tanto debe existir una demanda manifiesta -aunque haya otras latentes- por parte del paciente, de la familia o de las entidades o instituciones en la que aquella haya estado delegada.

Tiene por objetivo construir un proceso vital en las personas implicadas en la situación arteterapéutica y es por ello que el arteterapeuta debe huir de actitudes como las de querer estar al servicio, de ayudar: esto limita el reconocimiento de la propia posición subjetiva y a menudo es una forma omnipotente de relacionarse con el otro. No es un espacio para compartir entre arteterapeuta y paciente. Es una relación asimétrica - cada uno a partir de su proceso- pero no jerárquica. Podríamos denominarlo como una "diferenciación asistida" a partir de una "diferenciación implicada". No es tampoco un acompañamiento dirigido, donde lo que guiaría el desarrollo de la sesión serían las propias resonancias acerca de aquello que va aconteciendo. Es más bien un espacio para la escucha y la observación atenta y pausada, sin expectativas previas. Sin esperar respuestas. Sin espera. En atención abierta a la propuesta del otro. De como es en el otro y para el otro lo que él va promoviendo. Registrando y elaborando -en un momento posterior-, como nos implica, que resonancias -ahora sí- tiene en nosotros lo que va haciendo / aconteciendo en el otro. 
Eso es precisamente lo que facilita la libertad para ese especial inter-juego emocional en que se desarrolla en la sesión de Arteterapia.

El arteterapeuta deberá contemplar que sean las interrogaciones más que las certidumbres las que guíen su posición. Ello comporta necesariamente un "hacer caso" -en su vertiente humana y profesional- $a$ y con el otro.

Tendrá que representar la ley simbólica. Por lo tanto tendría que comprender que no es la ley simbólica, solo la representa, y ello porque la habrá interiorizado en base a su propio proceso terapéutico. De este modo la sesión será un lugar sin censura, donde las situaciones aparentemente negativas como las resistencias o las ausencias, serán tomadas como partes fundamentales del proceso. En la sesión no tendrá cabida la moral -entendida como lo que es bueno o malo, pertinente o no, etc.- sino la ética, aquella que acoge lo que presenta el paciente y permite el despliegue para su elaboración, con la única salvedad de aquello que cae fuera de los límites del marco, o sea la violencia - diferenciándola de la agresividad-y el sexo diferenciándolo de la sexualidad-.

Para ello el arteterapeuta habrá de tener cuidado con las interpretaciones, puesto que en demasiadas ocasiones la interpretación consiste en una proyección que borra la diferenciación entre el paciente y el arteterapeuta. Del mismo modo este último tendrá que valorar el alcance de su intervención técnica -al nivel de los lenguajes artísticos, los materiales y sus procedimientos- sobre el proceso, ya que no puede dejar de ser un mediador entre ellos y el paciente. Para ello habrá de ajustar sus propuestas para que el propio paciente encare sus producciones a partir de sus capacidades. Parafraseando a los minimalistas podemos decir aquello de "menos es más". Por supuesto esto también implica al arteterapeuta ya que este también debe ajustar las propuestas a sus capacidades, a lo que él puede sostener.

\section{COMPETENCIAS FUNDAMENTALES COMO ARTETERAPEUTA Y COMO INVESTIGADOR}

Partiendo de lo que podemos considerar aspectos fundamentales en el Arteterapia y la profesión del arteterapeuta, presentamos a continuación un conjunto de microcompetencias altamente interrelacionadas que completan el perfil profesional y humano, competencias que actúan como una realidad global y conforman la necesaria competencia profesional:

Competencia arteterapéutica

- Comprender la necesidad de establecer y mantener la relación arteterapéutica como un proceso creativo dentro de un marco sostenedor y asegurador.

- Saber articular la escucha y el desarrollo del vínculo entre arteterapeuta y paciente.

- Conocer las propias capacidades y límites.

- Conocer suficientemente sus propias formaciones psíquicas. 
- Comprender y sostener el compromiso que uno contrae aceptando estar en posición de arteterapeuta.

- Haber desarrollado un proceso psicoterapéutico personal.

- Vivenciar la transferencia y comprender la necesidad de supervisar la práctica profesional.

- Atender a la necesidad de formarse de manera continuada.

- Conocer que es el Arteterapia como práctica dentro del marco arteterapéutico diferenciándolo de otros planteamientos, tanto artísticos como psicoterapéuti$\cos$.

- Ser capaz de desarrollar un trabajo poético/simbólico/creativo, deslindándolo de aquello imaginario que pueda dificultarlo.

Competencia artística (desde el arte, a través del arte y por el arte)

- Interés por el arte y los lenguajes artísticos

- Poner en valor la realidad inclusiva del arte.

- Desarrollar actividades de producción en cualquiera forma de arte.

- Tener una concepción antropológica amplia y abierta de lo que contiene aglutina el concepto "arte" (arte, artes y oficios, cultura visual, arte infantil, gastronomía, indumentaria, prácticas estéticas antiguas y actuales....etc.).

Competencia cultural (intercultural)

- Conocimiento exploratorio sobre nuestras propias herencias culturales y nuestros sentimientos.

- Tener un Conocimiento amplio y sensible de las culturas y costumbres de las personas y de sus países de origen.

- Tener conciencia de como el trabajo arteterapéutico puede estar afectado por la cultura, edad, procedencia, género, creencias religiosas, etc.

- Conocer los parámetros de interacción sociales para participar en su transformación.

- Ser capaces de reflexionar sobre la propia cultura y conocer cómo esta influye en la forma en que nos vemos a nosotros mismos y a los demás.

- Capacidad para conocer, reconocer, contextualizar, valorar y ser capaces de celebrar y promover la diversidad antropológica, cultural, generacional, funcional, técnica y estilística de las manifestaciones y procesos arteterapéuticos.

- Conocer y valorar como la competencia cultural permite una visión humanizada de observar e investigar en el ámbito del Arteterapia.

Competencia crítica

- Capacidad para ejercer la profesión de manera crítica, autocrítica y reflexiva.

- Saber reconocer y ser sensible a las características de cada una de las personas a fin de ajustar la intervención, haciéndoles saber que son escuchados, respetados, valorados, y capaces de actualizar sus capacidades creativas. 
- Saber reconocer las desigualdades creadas por lo social la cultura (discriminaciones por razones de género, clase social, edad, cultura, capacidades físicas, etc.) y evitar actuaciones sesgadas o mediatizadas por estos parámetros.

- Tener conocimiento de los aspectos más relevantes y fundamentales de otras disciplinas como las artes, el psicoanálisis, la psiquiatría, la psicología del ciclo vital, etc...

Competencia ética

- Entender el Arteterapia como un proceso a desarrollar con el paciente y no para el paciente.

- Mantener la confidencialidad del trabajo tanto sobre lo hablado como sobre las producciones desarrolladas en las sesiones.

- Capacidad para respetar la dignidad de las personas participantes y proteger su bienestar.

- Compromiso ético sobre la confidencialidad de la información obtenida en las sesiones de trabajo y/o en las investigaciones.

- Comprender la necesidad de respetar los derechos, la dignidad, la autonomía y las dificultades de cada paciente.

Competencia comunicativa

- Aprender a trabajar tanto individualmente como en equipo. Conocer de ello tanto las oportunidades como sus dificultades.

- Conocer, comprender y valorar las manifestaciones perceptivas, estéticas, creativas, interpretativas, comunicativas, expresivas y representacionales que pueden estar presentes en las personas.

- Habilidad comunicativa tanto no verbal y verbal, como mediante imágenes u otras formas de representación, que permitan la comunicación adecuada para el desarrollo de la tarea.

- Valorar que la comunicación es imprescindible en el contacto entre las personas y que cualquier sistema comunicativo puede promover el crecimiento personal.

- Capacidad para informar adecuadamente de las acciones y procedimientos seguidos en las prácticas arteterapéuticas.

Competencia creativa

- Conocer y valorar los procesos que facilitan y desarrollan la creatividad en el trabajo arteterapéutico.

- Saber esperar, escuchar, accionar, perderse, encontrar soluciones e improvisar, adaptarse a situaciones nuevas, problemas nuevos, etc.

- Habilidad para promover y valorar procesos creativos a nivel individual y colectivo, mediante el desarrollo de percepciones no estereotipadas y del pensamiento divergente. 
Competencia Investigadora

- Conocer las metodologías científicas y la forma en que las investigaciones relacionadas con el Arteterapia se planifican, se desarrollan y se interpretan.

- Capacidad para utilizar los conceptos de validez y precisión, así como los procedimientos necesarios que permitan interpretar los resultados de los ensayos.

- Capacidad para confirmar o refutar hipótesis, validar intervenciones o clarificar hechos relativos a la práctica del Arteterapia, observando de forma sistemática y rigurosa las intervenciones.

- Conocer las metodologías de investigación científica categorizadas en los dos grandes enfoques; cualitativo y cuantitativo y desarrollar un pensamiento autónomo y reflexivo.

- Poseer capacidad interpretativa, crítica, reflexiva, propositiva y argumentativa para su desarrollo humano y profesional.

- Conocer y saber integrar en la investigación los principios éticos, las leyes y las normativas profesionales que rigen cualquier investigación.

- Habilidad para utilizar correctamente el marco arteterapéutico en la investigación, así como aplicar las propuestas metodológicas más acordes y que permitan avanzar en el conocimiento y los estudios a realizar.

- Saber informar adecuadamente a todos los sujetos relacionados con la investigación de aquello que les concierna.

- Compromiso ético en continuar su propia formación disciplinar y profesional.

\section{OBJETIVOS DE LA INVESTIGACIÓN EN ARTETERAPIA}

La investigación en Arteterapia tiene como principal objetivo la mejora de los resultados de la intervención arteterapéutica y a su vez incrementar los conocimientos y las mismas prácticas del Arteterapia otorgándoles el adecuado reconocimiento social. Como en cualquier disciplina, el avance en el conocimiento es una tarea que corresponde al propio colectivo de arteterapeutas que deben necesariamente implicarse a partir de una posición ética, según sus propias capacidades y las circunstancias o entornos donde intervienen. Por otra parte cabe defender que estos avances han de ser divulgados y compartidos para que ejerzan su principal función que es, en primer lugar, el beneficio que las personas pueden obtener del Arteterapia, la mejora permanente de las prácticas desarrolladas y el conocimiento de los resultados como forma de acrecentar el conocimiento de la profesión desde un posicionamiento de evaluación y de retroalimentación permanente.

Como en cualquier disciplina y para cualquier profesional, se percibe la investigación como una tarea compleja para la que hace falta tener un especial sentido de la curiosidad, derivada tanto del propio ejercicio profesional como del objeto sobre el que el Arteterapia es competente, el desarrollo emocional de las personas y sus capacidades creativas. Por otra parte la complejidad de las intervenciones y el pensar que para ello ya existen científicos que basan toda su razón de ser en la investi- 
gación, separando de ese modo, las prácticas arteterapéuticas de su necesaria actualización. Por ello es necesario desarrollar la conciencia de fundamentar las prácticas, bien atendiendo a investigaciones ya desarrolladas, bien participando en otras, y así poder divulgar los nuevos conocimientos. Implementando una o varias teorías que aún hoy han de conceptualizarse y desarrollarse y experimentando con formas de intervención que permitan nuevos caminos. Todo ello ayudará a fortalecer y consolidar la profesión en el ámbito social.

Los ámbitos de educación superior que desarrollan la formación profesionalizadora en Arteterapia por vía de Postgrados, tienen la responsabilidad de generar procesos de investigación y formar en dinámicas y metodologías de investigación acordes al objeto y a la práctica del Arteterapia. Prácticas que deben posicionarse teniendo en cuenta los parámetros científicos resultantes de las investigaciones desarrolladas en Arteterapia o en metodologías afines a su objeto. Objetivo imprescindible para que las y los estudiantes pasen de ser meros consumidores a ser productores de conocimiento y de esta forma corresponsables de los avances en la disciplina.

\section{ELEMENTOS Y ESPACIOS PARA LA INVESTIGACIÓN}

Asumir que los procesos de investigación nos permitirán avanzar en el conocimiento de la disciplina nos lleva a cuestionar y determinar en que ámbitos podemos desarrollar indagaciones que permitan aportar nuevos y significativos elementos al conocimiento, que puedan ayudar a mejorar la práctica del Arteterapia y asimismo decidir cuales son los métodos de investigación adecuados y cuales pueden ser las perspectivas en la que desarrollar nuestra propuesta. Podemos cuantificar los hechos pero no así los significados que solo pueden atenderse desde una perspectiva cualitativa. Dos perspectivas, cualitativa/cuantitativa que deben participar del objeto de la investigación en su justa medida. Atendiendo la tipología de los datos prevalecerá una u otra, poniendo en consideración que el principal objetivo de la ciencia es la adquisición de conocimientos y la adecuación ética de los mismos al objeto del estudio, por lo tanto es fundamental la elección del método adecuado que ayude a conocer esa realidad.

Un cierto debate sobre la conveniencia de uno u otro paradigma metodológico recorre los ámbitos de la investigación. Por razones obvias no es posible renunciar a priori a ninguna de las dos perspectivas, cada una de ellas ocupa un importante espacio en los ámbitos de la investigación científica y cada una puede aportar datos relevantes que serán hasta complementarios en algunos de los proyectos. Para responder a los interrogantes de nuestras investigaciones, disponemos de estos dos grandes paradigmas el cuantitativo y el cualitativo, que utilizaremos en función de la naturaleza de los datos y atendiendo a que estos son únicamente herramientas y no la finalidad del estudio. 
Las ciencias sociales y asimismo el ámbito del Arteterapia disponen de una gran variedad de métodos de investigación y aunque responden a criterios muy diversos, todos pueden resultar útiles en nuestra tarea investigadora. A continuación presentamos brevemente algunos de esos métodos, todos ellos enmarcados en el paradigma cualitativo. Nuestra intención no es ser exhaustivos ni mostrar la globalidad de las metodologías y clasificaciones existentes. Esta muestra se presenta solo a modo de ejemplo para iluminar nuestro discurso.

La razón de nuestra elección responde a la habitual utilización de estos métodos cualitativos porque centran su atención sobre los fenómenos, estudiando y registrando los datos de forma narrativa y dando importancia al sujeto y a las fuentes documentales, de forma que es posible obtener diversas interpretaciones de los datos y de la realidad. Algunas de las tipologías con la que realizar nuestras investigaciones serían: la investigación acción, las historias de vida o métodos biográficos, los estudios de caso, las etnografías, la teoría fundamentada, etc., Será la intencionalidad del investigador junto a la adecuación al objeto del estudio la que determine cual de las tipologías será más ajustado aplicar.

\section{Investigación acción}

Es una tipología que complementa las acciones experimentales de investigación con otras de tipo social para así responder a problemas y a retos sociales. Por esta razón se considera que es una tipología de investigación que permite que tanto los avances en el conocimiento como los cambios sociales, se produzcan en paralelo.

\section{Historias de vida o métodos biográficos}

Investigaciones de carácter narrativo en las que se reúnen vivencias, sucesos, acontecimientos significativos en la vida de los sujetos, permitiendo ordenar, relacionar y contrastar los datos alrededor de líneas argumentales que permiten lecturas coherentes de los hechos. Para construir una historia de vida nos apoyamos en autobiografias, biografias o relatos de personas, atendiendo a las voces únicas de cada individuo.

\section{Estudios de caso}

Se trata de un método de investigación que pretende comprender un caso, sin la finalidad última de la comprensión de un conjunto de casos o un problema global. Se utilizan para analizar y entender las realidades sociales, centrando su estrategia en procesos de indagación que examinan, analizan y permiten reflexionar sobre fenómenos contemporáneos en contextos reales, atendiendo de forma sistemática la información que permite conocer en profundidad esos fenómenos.

\section{Etnografías}

Las etnografías tienen su origen en la antropología cultural y se basan en fórmulas en las que la recogida de datos se realiza desde la perspectiva de observación participante, el investigador participa en algunos momentos cuotidianos de los sujetos, observa lo que sucede, escucha y se interroga. El investigador esta situado cerca 
de la fuente de información, donde la acción se produce, atendiendo y aprendiendo de los mundos simbólicos y de los lenguajes de los sujetos que estudia.

\section{Teoría fundamentada}

Es una alternativa para la definición de nuevas teorías a partir del análisis de datos sin fundamentar estos logros en la comprobación y contrastación con las teorías existentes. Sus logros son formulaciones teóricas de la propia realidad, una especial y continua forma de obtener, revisar, comparar y contrastar esa realidad y así pensar y conceptualizar los datos obtenidos. La recogida de datos se realiza mediante la interacción humana (entrevistas, observación participante, etc.,) y su análisis pretende iluminar los procesos que emergen de esa interacción.

\section{Método observacional}

Aunque la observación es la herramienta para la comprobación de las hipótesis de toda investigación, entendemos como método observacional aquel en el que se verifican las hipótesis o se recopilan los datos sin introducir variables, modificar o controlar la muestra. El interés de la investigación es observar y conocer lo que se realiza de forma espontanea, las acciones y conductas que de forma natural realizan los sujetos.

\section{Fenomenología}

Es una metodología que pretende describir significados a partir de las experiencias vitales y cotidianas de los sujetos, vivencias subjetivas de quienes las han vivido. Se entiende el mundo como inconcluso, en permanente construcción por la intervención de los sujetos que lo modifican y le imprimen significado.

\section{CONSTRUYENDO UN PARADIGMA DE FUTURO}

Partimos de la convicción de que el presente y el futuro del Arteterapia dependen de la formación profesional y humana del arteterapeuta, que incluye, de manera significativa, su capacidad investigadora y la consciencia asumida en pro de la producción científica de conocimientos. Asumiendo esa convicción, es deseable que las y los profesionales posean y estén habituados a ejercer procesos de razonamiento mediados por la observación, interrogarse por sus propias acciones profesionales, dudar de los recursos utilizados habitualmente y de los conocimientos comunes, saborear los conocimientos contenidos en los referentes bibliográficos y desarrollar la intuición hacia nuevos paradigmas y procesos de indagación, entre otras habilidades. Espíritus atentos, críticos, permanentemente interesados por avanzar en su disciplina e intelectualmente productivos. Profesionales que entienden que la investigación los incluye como sujetos y al mismo tiempo como objetos de su intervención.

Así la investigación no es una materia más para adornar el curriculum, sino una obligación ética por parte de todos aquellos profesionales comprometidos en el ejercicio de la práctica del Arteterapia. Asumir ese rol profesional como investiga- 
dor no es una tarea fácil, debe construirse rompiendo aquellas barreras que inhiben al arteterapeuta para crecer intelectual y humanamente, paradigmas prestablecidos que llevan a:

- No interrogarse a partir de su práctica, dando por valido todo aquello que proviene de la formación.

- Cierto conformismo intelectual

- No cuestionar su propia posición subjetiva como interviniente dentro de su práctica

- No validación del error como recurso del aprendizaje

- Escaso sentido critico y reflexivo

- No reconocimiento como generadores de ciencia

La idiosincrasia de una profesión que desarrolla sus prácticas de forma individual -aunque se trabaje dentro de un equipo-, hace necesario configurar proyectos de investigación sustentados en redes de investigadores y/o en equipos de trabajo para que las ideas y las hipótesis individuales se catalicen y conceptualicen en el seno del equipo y que permitan nuevos conocimientos sustentados en el dialogo, la discusión y la construcción compartida de conocimientos científicos y no solo reservados a la atención del profesional o de reducidos colectivos.

\section{REFERENCIAS BIBLIOGRÁFICAS}

BERICAT, E. (1998). La integración de los métodos cuantitativo y cualitativo en la investigación social: Significado y Medida. Barcelona. Editorial Ariel.

DELGADO, J. M. Y GUTIÉRREZ, J. (1995). Métodos y técnicas cualitativas de investigación en ciencias sociales. Madrid. Editorial Síntesis.

GREEN, A. (2007) Jugar con Winnicott. Buenos Aires. Ed. Amorrortu.

IZUEL, M. (2005) "De la transferencia al vínculo: oportunidad de la ficción en los procesos terapéuticos". Cuadernos de Psicomotricidad n ${ }^{\circ}$ 29. p. 15-22. Guipuzkoa. Escuela de Psicomotricidad de la UNED.

IZUEL, M. (2009) "Entre los desfiladeros de lo imaginario y lo simbólico, la búsqueda del autor". Encuentros con la expresión, $n^{\circ}$ 2. p. 13-23. Murcia. Mancomunidad del valle del Ricote.

IZUEL, M. (2011) "El dispositivo grupal en la formación personal de arteterapeutas". Arteterapia: papeles de arteterapia y educación artística para la inclusión social, $n^{\circ}$ 6. p. 33-49. Madrid. Universidad Complutense de Madrid.

TAYLOR, S.; BOGDAN, R. (2002). Introducción a los métodos cualitativos de investigación. Barcelona. Paidós.

VALLÈS, J. (2005) Competencia multicultural en educación artística. Contextos y perspectivas de futuro en la formación de las maestras y los maestros. Tesis doctoral. Girona. Universitat de Girona. 
VALLÈS, J.; ÁLVAREZ, D.; RICKENMANN, R. (2011) L'Activitat docent, Intervenció, Innovació, Investigació. Girona. Documenta Universitaria i Edicions a Petició, S.L.

VALLÈS, J.; CALBÓ, M. (2011) "Competència Cultural i Artistica". Revista catalana de pedagogía 7. p. 81-98. Barcelona. IEC.

WINNICOTT, D. W. (1996) Realidad y Juego. Barcelona. Ed. Gedisa. 